\title{
Mid-term outcomes of bony increased offset-reverse total shoulder arthroplasty in the Asian population
}

\author{
Kirtan Tankshali, Dong-Whan Suh, Jong-Hun Ji, Chang-Yeon Kim \\ Department of Orthopedic Surgery, Daejeon St. Mary's Hospital, College of Medicine, The Catholic University of Korea, Daejeon, Korea
}

\begin{abstract}
Background: To evaluate clinical and radiological outcomes of bony increased offset-reverse total shoulder arthroplasty (BIO-RSA) in the Asian population at mid-term follow-up.

Methods: From June 2012 to August 2017 at a single center, 43 patients underwent BIO-RSA, and 38 patients with minimum 2 years follow-up were enrolled. We evaluated the clinical and radiological outcomes, and complications at the last follow-up. In addition, we divided these patients into notching and no-notching groups and compared the demographics, preoperative, and postoperative characteristics of patients.

Results: Visual analogue scale, American Shoulder and Elbow Surgeons, University of California-Los Angeles Shoulder Scale, and Simple Shoulder Test scores improved significantly from preoperative $(5.00,3.93,1.72,3.94)$ to postoperative $(1.72,78.91,28.34,7.66)(p<0.05)$ outcomes. All range of motion except internal rotation improved significantly at the final follow-up ( $\mathrm{p}<0.05$ ), and the bone graft was well-incorporated with the native glenoid in all patients (100\%). However, scapular notching was observed in 20 of 38 patients (53\%). In the comparison between notching and no-notching groups (18 vs. 20 patients), there were no significant differences in demographics, radiological parameters, and clinical outcomes except acromion-greater tuberosity (AT) distance ( $\mathrm{p}=0.003$ ). Intraoperative complications included three metaphyseal fractures and one inferior screw malposition. Postoperative complications included ectopic ossification, scapular neck stress fracture, humeral stem relaxation, and late infection in one case each.

Conclusions: BIO-RSA showed improved clinical outcomes at mid-term follow-up in Asian population. However, we observed higher scapular notching compared to the previous studies. In addition, adequate glenoid lateralization with appropriate humeral lengthening (AT distance) might reduce scapular notching.
\end{abstract}

Keywords: Bony increased offset-reverse total shoulder arthroplasty; Scapular notching; Graft incorporation; Complications; Glenoid lateralization

\section{INTRODUCTION}

Reverse total shoulder arthroplasty (RSA) is a relatively new development in the field of shoulder surgery and is undergoing constant refinement. However, there is no consensus on the single best implant design or technical aspects of the procedure. The history of RSA dates to the 1970s when this concept of reversing was proposed for the first time [1]. There are many modifications to the

Received: February 14, $2021 \quad$ Revised: May 2, $2021 \quad$ Accepted: May 5, 2021

Correspondence to: Jong-Hun Ji

Department of Orthopedic Surgery, Daejeon St. Mary's Hospital, College of Medicine, The Catholic University of Korea, 64 Daeheung-ro, Jung-gu, Daejeon 34943, Korea

Tel: +82-42-220-9530, Fax: +82-42-221-0429, E-mail: jijh87@gmail.com, ORCID: https://orcid.org/0000-0003-1087-3254

Financial support: None.

Conflict of interest: None.

Copyright(C) 2021 Korean Shoulder and Elbow Society.

This is an Open Access article distributed under the terms of the Creative Commons Attribution Non-Commercial License (http://creativecommons.org/licenses/by-nc/4.0/) which permits unrestricted non-commercial use, distribution, and reproduction in any medium, provided the original work is properly cited. 
initial design, but none have been accepted to date as the implant of choice. RSA designs are primarily based on the pioneering work of Grammont who first proposed the concept of medialization and distalization of the center of prosthesis rotation [2]. Nevertheless, this prosthesis exhibited design-related complications such as scapular notching, instability, and aseptic loosening on long-term follow-up, requiring further refinement. While instability is a complication that can be addressed using thicker poly-liner or metallic humeral spacers, which will achieve greater deltoid tension and better balancing of the prosthesis, aseptic loosening has been shown to be reduced with uncemented stem implantation and remains more of a complication of cemented stems [3]. However, notching remains the most common complication that requires surgical technique modification or implant design modification or both and has yet to be addressed. Inferior overhang of the glenosphere, inferior tilting of the glenosphere, lateralization of the center of the prosthesis rotation by increasing glenosphere arc, and metal augmentation of the glenosphere are described to prevent scapular notching $[4,5]$.

However, these techniques have drawbacks. Inferior tilting of the glenosphere can increase tension on the deltoid and has been shown to reduce the longevity of the implant [6,7]. Increasing the glenosphere arc or adding metal augments shifts the center of prosthesis rotation within the glenoid component and increases the unbalanced load on the glenoid component with each movement, which can lead to early glenoid component loosening [8]. As an alternative to these techniques, bony increased offset-RSA (BIO-RSA) was proposed to use autograft harvested from the patient's humeral head to lateralize the glenoid baseplate. This technique has been proposed to maintain the center of rotation at the bone implant interface, providing dual benefits of lateralizing the center of rotation to reduce scapular notching and improvement in rotations, while also preventing unbalanced loading of the glenoid component [9]. However, controversy remains about development of notching after BIO-RSA (Fig. 1).

The purpose of this study was to evaluate the clinical and radiological outcomes of BIO-RSA in the Asian population at the mid-term follow-up. We hypothesized that BIO-RSA would lead to improved clinical outcomes in indicated patients along with less scapular notching and postoperative complications.

\section{METHODS}

This study was approved by the Institutional Review Board of Daejeon St. Mary's Hospital (IRB No. C17RESI0067). Informed consent was obtained from the patients included in this study.

This is a retrospective study of 38 patients who underwent RSA using the bony increased offset technique (BIO-RSA) from
June 2012 to August 2017 at a single center. All the patients presented during the study period with cuff tear arthropathy, massive retracted irreparable cuff tear (Patte stage 3 or Goutallier stage 3/4), primary/secondary glenohumeral osteoarthritis with massive cuff tear, recurrent shoulder dislocation with massive cuff tear, or glenoid fracture sequela were included in the study. We excluded acute proximal humerus fracture, avascular necrosis, and revision RSA cases. In acute proximal humerus fracture, BIO-RSA was not attempted as the primary treatment because of concerns of the non-availability of adequate humeral head bone stock for autograft harvesting. In addition, patients younger than 65 with massive cuff tears received an alternate form of treatment (partial repair or superior capsule reconstruction) and were not included in the study. We also operated on three revision RSA cases during the study period (two humeral head allografts and one iliac autograft) with BIO-RSA. However, patients who received revision surgery were not included in the study. All the patients were operated on by a single surgeon, the senior author of this study. A 36-mm glenosphere and 25-mm-diameter glenoid baseplate with $25-\mathrm{mm}$ peg length was used without inferior tilting. For the humeral stem, we used the cemented stem in 30 patients and uncemented stem in eight patients.

Glenohumeral arthritis along with cuff retraction and fatty infiltration (Goutallier stage) was evaluated in all patients preoperatively. Glenoid morphology was classified according to the types described by Walch et al. [10] to aid in planning and positioning of the glenoid component intraoperatively. Postoperatively, all patients were followed for a minimum of 2 years (mean, 3 years). The average follow-up period was $36.7 \pm 16$ months (range, 2478 months). Postoperative outcomes were evaluated with radiographs, clinical scores, and range of motion evaluations. Radiographic follow-ups included three monthly shoulder X-rays during the first year after operation and yearly X-rays thereafter. We evaluated scapular notching, signs of osteolysis, and implant
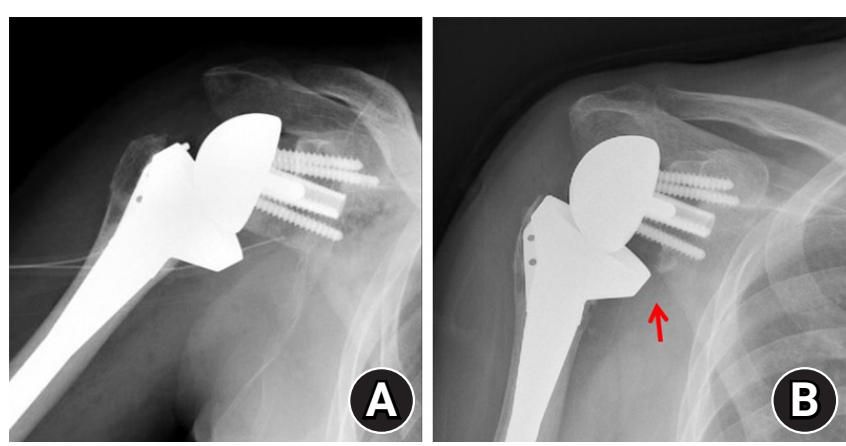

Fig. 1. (A) Immediate X-ray showing graft placement on the glenoid. (B) Two-year follow-up X-ray showing grade 3 scapular notching (arrow). 
(glenoid and humeral components) loosening. Scapular notching was classified according to the Nerot-Sirveaux classification at the final follow-up. Specifically, we evaluated postoperative radiologic parameters including peg-glenoid rim distance (PGRD), prosthesis-scapular neck angle (PSNA), acromion-greater tuberosity (AT) distance, and glenoid-greater tuberosity (GT) distance. PGRD was measured to assess the inferior positioning of the baseplate, PSNA to assess the inferior tilt, AT distance to assess the amount of humeral lengthening, and GT distance to assess the amount of medialization or lateralization of the humerus. The clinical outcome evaluation included American Shoulder and Elbow Surgeons (ASES), University of California-Los Angeles (UCLA), Simple Shoulder Test (SST), and visual analog scale (VAS) scores along with range of motion evaluation. Finally, we divided patients into notching and not-notching groups and compared the demographics, preoperative, and postoperative characteristics of the patients between the two groups.

\section{Surgical Technique}

All the patients were operated in the beach chair position under general anesthesia supplemented with interscalene block. The deltopectoral approach was used in all the cases. After identifying the cephalic vein and retracting it laterally, the plane was developed between the deltoid and pectoralis major muscles. Upper fibers of the pectoralis major tendon were released from its attachment site, leaving approximately $5 \mathrm{~mm}$ of stump laterally to repair at the end of the procedure. Next, the clavipectoral fascia was incised, and the conjoined tendon was identified and retracted medially. The subscapularis and identified vessels running across its inferior margin were ligated. Next, the biceps long head tendon was tagged and tenotomized, and then the subscapularis was tagged and removed from its humeral attachment at the lesser tuberosity. The humeral head was dislocated, and a humeral cut was performed using the standard preparation guide with $20^{\circ}$ retroversion. A cut guide was placed over the graft, and a saw blade was used to create a 7-mm or 10-mm grafting area. We always harvested an autograft of 7-mm thickness from the excised humeral head because autografts $10 \mathrm{~mm}$ in thickness created too much tension in the shoulder joint during reduction of the prosthesis. The glenoid was prepared in standard fashion with minimum reaming up to the subchondral bone. The harvested graft was fitted in the 25 -mm-diameter glenoid baseplate with $25-\mathrm{mm}$ peg length and was inserted in the predrilled peg hole without inferior tilting (neutral) (Fig. 2) Usually, the baseplate was fixed with four screws, and then the $36-\mathrm{mm}$ glenosphere was implanted. Next, the humeral stem was prepared in standard fashion with the stem guide and $20^{\circ}$ retroversion. Trial stems were inserted, and the tension and stability were measured before final humeral stem implantation. Trans-osseous holes were drilled followed by the passage of No. \#2 ethibond from the holes before the final stem insertion for subscapularis reattachment. The humeral stem was inserted, the tension was measured again with trial liner, and the subscapularis tendon was reattached. This was followed by liner insertion as per the trial size and reduction of the prosthesis. Two drains were inserted to reduce fluid or hematoma collection in the dead space followed by wound closure in layers.

Postoperatively, we immobilized all the patients in an abduction brace for 6 weeks. Only elbow, hand, and wrist mobilization were allowed along with pendulum exercises. At 6 weeks, the brace was weaned off and gradual mobilization was initiated which was continued for 3 months postoperatively. After 3 months, gradual strengthening exercise programs were adopted.
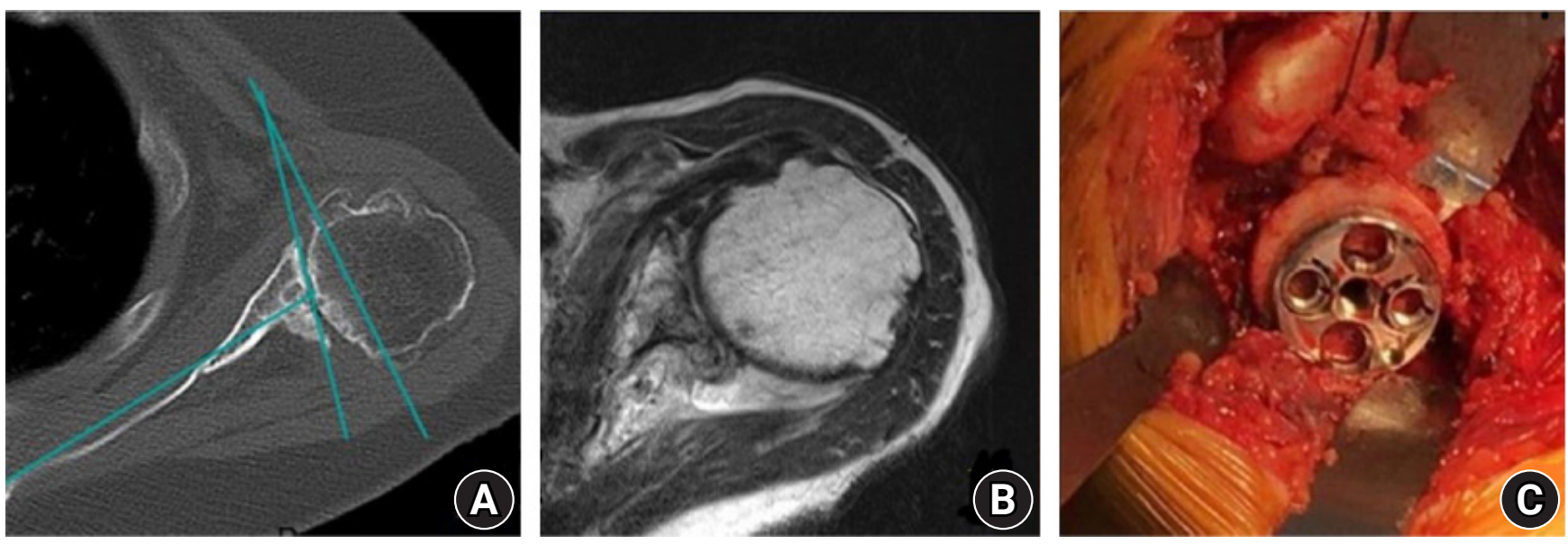

Fig. 2. Preoperative axial computed tomography scan (A) and magnetic resonance imaging (B) showing type C glenoid (C) with intraoperative placement of an autograft on the glenoid baseplate. Preoperative mid-axial computed tomography showed the glenoid version (glenoid version is calculated as the angle between the glenoid line and the line perpendicular to the scapular axis). 


\section{Statistical Analysis}

A p-value $<0.05$ was defined as statistically significant. All statistical analyses were performed using the IBM SPSS ver. 20.0 (IBM Corp., Armonk, NY, USA). Measurements were expressed as mean \pm standard deviation with a confidence interval of $95 \%$ for continuous variables. To determine the impact of intervention, we used the paired t-test to compare postoperative with preoperative measurements. Internal rotation was scored as per the level of vertebrae. Starting from Sacrum as 0 each lumbar vertebra was given score +2 (ie L5-2, L4-4....L1-10). T12 was scored as 12 and subsequent thoracic vertebra was scored +1 (ie T11-13, T1014...T3-21).

Table 1. Patient demographics and etiology of our study

\begin{tabular}{lc}
\hline Parameter & Value \\
\hline Age (yr) & $72.9 \pm 5.6$ \\
Sex (male:female) & $9: 29$ \\
Body mass index $\left(\mathrm{kg} / \mathrm{m}^{2}\right)$ & $24.8 \pm 3.2$ \\
Follow-up period $(\mathrm{mo})$ & $36.7 \pm 16(24-78)$ \\
Etiology & 22 \\
$\quad$ Cuff tear arthropathy & 3 \\
Recurrent shoulder dislocation due to & \\
$\quad$ massive cuff tear & 2 \\
Osteoarthritis with poor quality cuff & 10 \\
Isolated massive irreparable cuff tear & 1 \\
Glenoid fracture sequelae & \\
Glenoid type (Walch classification) & 24 \\
A1 & 3 \\
A2 & 7 \\
B1 & 3 \\
B2 & 1 \\
C
\end{tabular}

Values are presented as mean \pm standard deviation, number of patients, or mean \pm standard deviation (range).

\section{RESULTS}

There were 9 males and 29 females in our study, with an average age of $72.9 \pm 5.6$ years (range, $64-87$ years). The average BMI of the patients was $24.8 \mathrm{~kg} / \mathrm{m}^{2}$ (range, $18.8-33.2 \mathrm{~kg} / \mathrm{m}^{2}$ ) (Table 1 ).

According to the Hamada classification [11], there were six patients with grade $4 \mathrm{~B}$, five patients with grade $4 \mathrm{~A}$, nine patients with grade 3, 10 patients with grade 2, and eight patients with grade 1 arthropathy. Grade 1 patients either had a history of recurrent dislocation or had isolated massive irreparable cuff tear. As per the study by Walch et al. [10], there were 24 patients with $\mathrm{A} 1$, three patients with $\mathrm{A} 2$, seven patients with $\mathrm{B} 1$, and three patients with $\mathrm{B} 2$ types of glenoid and one patient with type $\mathrm{C}$ glenoid. Improvement in clinical parameters with p-values are shown in the table below (Table 2). All the scores, namely ASES, UCLA, SST, and VAS, improved significantly postoperatively compared to the preoperative status. In addition, active forward flexion, abduction, and external rotation improved significantly compared to the preoperative status $(\mathrm{p}<0.05)$. Internal rotation also exhibited improvement compared with preoperative scoring. However, this improvement was not significant $(\mathrm{p}=0.138)$. Radiolucent lines around the humeral stem were observed in 16 of 38 patients $(42.11 \%)$. However, only one patient developed stem loosening at 3 years follow-up. Glenoid graft was incorporated in all the patients (100\%). Scapular notching was observed in 20 of

Table 3. Notching at the 2-year follow-up

\begin{tabular}{lc}
\hline Scapular notching grade & Two-year follow-up \\
\hline 0 (No notching) & 18 \\
1 & 9 \\
2 & 9 \\
3 & 2 \\
4 & 0 \\
\hline
\end{tabular}

Table 2. Clinical scores and range of motion with p-value

\begin{tabular}{lccc}
\hline Parameter & Preoperative & Postoperative & p-value \\
\hline VAS score & $5.00 \pm 1.66$ & $1.72 \pm 1.28$ & NS \\
ASES score & $3.93 \pm 2.58$ & $78.91 \pm 11.83$ & NS \\
UCLA score & $1.72 \pm 1.28$ & $28.34 \pm 3.34$ & NS \\
SST score & $3.94 \pm 3.50$ & $7.66 \pm 2.02$ & NS \\
Range of motion $\left(^{\circ}\right)$ & & & \\
$\quad$ Forward elevation & $101.67 \pm 35.65$ & $134.86 \pm 18.28$ & NS \\
$\quad$ Abduction & $93.65 \pm 37.17$ & $132.84 \pm 19.74$ & NS \\
$\quad$ External rotation & $22.30 \pm 12.78$ & $31.35 \pm 11.47$ & NS \\
$\quad$ Internal rotation & $5.81 \pm 5.51$ & $7.32 \pm 3.92$ & 0.138 \\
\hline
\end{tabular}

Values are presented as mean \pm standard deviation.

VAS: visual analog scale, ASES: American Shoulder and Elbow Surgeons, UCLA: University of California-Los Angeles, SST: Simple Shoulder Test, NS: not significant. 
38 patients (53\%), and the grading distribution is shown below (Table 3). In the comparison between notching and not-notching groups, there were no significant differences between the two groups in demographics, radiological parameters, and clinical outcomes except AT distance $(p=0.003)$ (Table 4). In addition, the PSNA, PGRD, and GT distance exhibited no significant differences between the two groups $(\mathrm{p}=0.940)$.

Intraoperative complications of our study included one inferior screw malpositioning and three humeral metaphyseal fractures, which were treated with simple wiring. Postoperative complications observed were one heterotopic ossification (1.5 months), one scapula neck fracture (27 months) (Fig. 3), one humeral stem loosening, and one late infection. The humeral stem loosening patient presented with occasional pain (VAS 2) and restriction of motion (forward flexion 110, abduction 110, external rotation 20, and internal rotation L4). However, she refused to
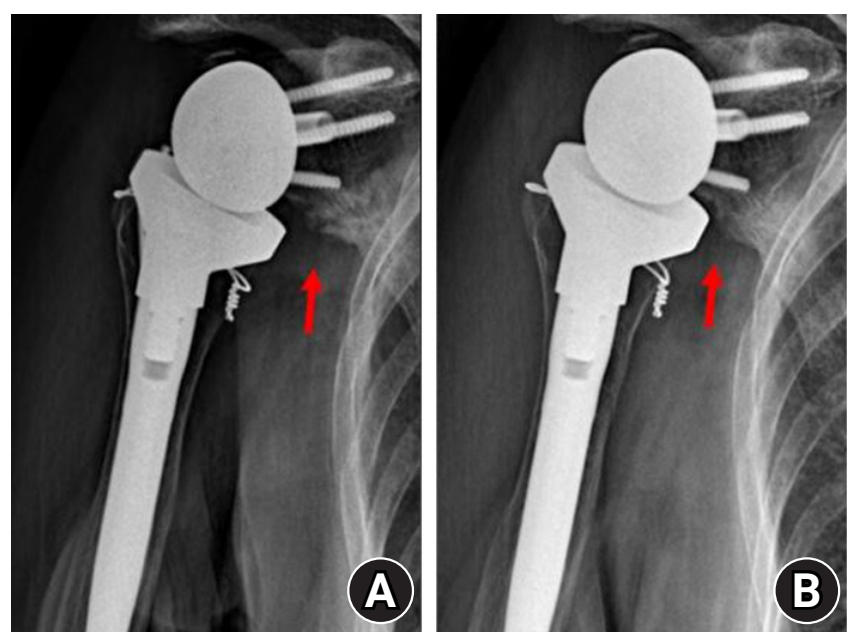

Fig. 3. (A) Scapular neck stress fracture (arrow) developed after trivial trauma. (B) Callus formation (arrow) observed at 3 months with conservative treatment. undergo revision surgery (Fig. 4). The infection case presented at

Table 4. Comparisons between the no-notching and notching groups in our study

\begin{tabular}{|c|c|c|c|}
\hline Variable & No-notching group $(\mathrm{n}=18)$ & Notching group $(\mathrm{n}=20)$ & p-value \\
\hline Age (yr) & $71.8 \pm 5.2$ & $73.9 \pm 5.9$ & 0.273 \\
\hline Sex (male:female) & $4: 14$ & $5: 15$ & 0.943 \\
\hline BMI $\left(\mathrm{kg} / \mathrm{m}^{2}\right)$ & $24.9 \pm 3.6$ & $24.6 \pm 2.9$ & 0.771 \\
\hline $\operatorname{PSNA}\left({ }^{\circ}\right)$ & $109.9 \pm 20.9$ & $104.5 \pm 15.1$ & 0.541 \\
\hline PGRD (mm) & $21.5 \pm 4.7$ & $24.6 \pm 9.4$ & 0.223 \\
\hline AT distance (mm) & $39.9 \pm 7.3$ & $47.7 \pm 7.2$ & 0.003 \\
\hline GT distance $(\mathrm{mm})$ & $44.7 \pm 7.6$ & $44.5 \pm 6.5$ & 0.940 \\
\hline \multicolumn{4}{|l|}{ Preoperative } \\
\hline VAS & $5.1 \pm 1.4$ & $4.9 \pm 1.8$ & 0.840 \\
\hline ASES & $44.0 \pm 23.1$ & $34.1 \pm 21.3$ & 0.840 \\
\hline UCLA & $18.5 \pm 7.0$ & $13.7 \pm 7.5$ & 0.057 \\
\hline SST & $4.2 \pm 2.9$ & $3.5 \pm 2.3$ & 0.452 \\
\hline \multicolumn{4}{|l|}{ Postoperative } \\
\hline VAS & $1.6 \pm 0.7$ & $1.2 \pm 0.8$ & 0.055 \\
\hline ASES & $78.2 \pm 12.2$ & $80.1 \pm 11.7$ & 0.642 \\
\hline UCLA & $28.6 \pm 3.6$ & $28.2 \pm 3.2$ & 0.738 \\
\hline SST & $7.4 \pm 2.3$ & $8.3 \pm 1.5$ & 0.134 \\
\hline \multicolumn{4}{|l|}{ Preoperative } \\
\hline Forward flexion $\left({ }^{\circ}\right)$ & $100.6 \pm 27.6$ & $105.0 \pm 38.9$ & 0.693 \\
\hline Abduction $\left({ }^{\circ}\right)$ & $91.4 \pm 33.9$ & $95.8 \pm 40.8$ & 0.724 \\
\hline External rotation $\left({ }^{\circ}\right)$ & $21.9 \pm 11.5$ & $22.6 \pm 14.2$ & 0.873 \\
\hline Internal rotation & Lumbar vertebrae 3 (sacrum-T7) & Lumbar vertebrae 3 (sacrum-T7) & 0.797 \\
\hline \multicolumn{4}{|l|}{ Postoperative } \\
\hline Forward flexion $\left({ }^{\circ}\right)$ & $133.9 \pm 17.2$ & $135.8 \pm 20.4$ & 0.757 \\
\hline Abduction $\left({ }^{\circ}\right)$ & $128.6 \pm 18.7$ & $136.8 \pm 20.4$ & 0.209 \\
\hline External rotation $\left({ }^{\circ}\right)$ & $29.7 \pm 10.2$ & $32.9 \pm 12.6$ & 0.408 \\
\hline Internal rotation & Lumbar vertebrae 2 (L5-T7) & Lumbar vertebrae 2 (L5-T8) & 0.816 \\
\hline
\end{tabular}

Values are presented as mean \pm standard deviation.

BMI: body mass index, PSNA: prosthesis-scapular neck angle, PGRD: peg-glenoid rim distance, AT: acromion-greater tuberosity, GT: glenoid-greater tuberosity, VAS: visual analog scale, ASES: American Shoulder and Elbow Surgeons, UCLA: University of California-Los Angeles; SST: Simple Shoulder Test. 
33 months after the primary procedure (RSA) and was treated with implant removal and prosthesis of antibiotic-loaded acrylic cement (PROSTALAC) insertion (Fig. 5).

\section{DISCUSSION}

Our study showed that, in Asian populations with a minimum of 2-year follow-up, BIO-RSA exhibited significantly improved clinical outcomes and range of motion except for that of internal rotation. The overall rate of notching was 53\% (20/38). However, there was no case of grade 4 glenoid notching, and $100 \%$ graft incorporation and no glenoid loosening were observed at the last follow-up. In the comparison between the "notching" and "no-notching" groups,
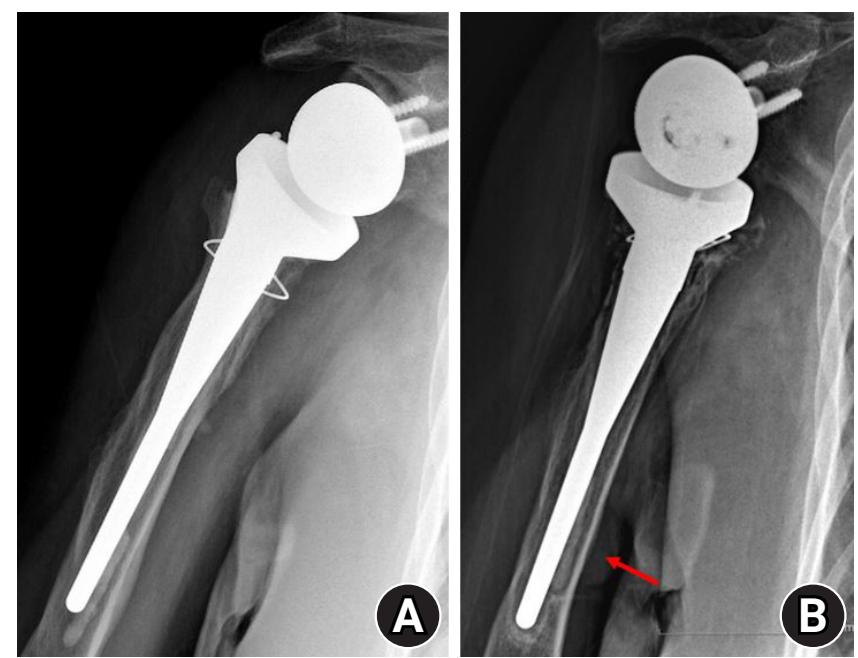

Fig. 4. (A) Immediate postoperative X-ray. (B) Aseptic loosening of the cemented humeral stem (arrow). there were no significant differences in demographics, radiological parameters, and clinical outcomes except AT distance.

Previously, few studies have reported outcomes of BIO-RSA. This is the first study reporting BIO-RSA outcomes in the Asian population. Conventional RSA is known to produce scapular notching, exhibit less improvement in rotational movements, impinge on free movements, and result in poor cosmesis due to medialization of the center of rotation [12]. Boileau et al. [9] originally described the method of BIO-RSA in 2011 to solve these issues. Unfortunately, since then, not many clinicians have adopted this technique. According to their results, the humeral autograft incorporated completely in $98 \%$ of cases (41/42) and partially in one. At a mean of 28 months postoperatively, no graft resorption, glenoid loosening, or postoperative instability was observed. We also observed $100 \%$ graft incorporation with no glenoid loosening or instability. In their study, significantly increased active anterior elevation and external rotation were noted at the final follow-up, similar to our findings. They reported inferior scapular notching in 19\% (8/42) and 86\% (36/42) of patients in their series who were able to internally rotate sufficiently to reach their back over the sacrum. Although internal rotation improvement was observed in our study similar to that in their study, with 37 of 38 patients being able to internally rotate to reach above the sacrum, the rate of notching was 20 of 38 (53\%), which is much higher than their reported rate. However, this rate of notching (53\%) is less than the reported rates of notching with conventional RSA (>63\%) $[2,13]$.

There have been few studies reporting on BIO-RSA outcomes, but their reported results are not similar. Kirzner et al. [14] pub-
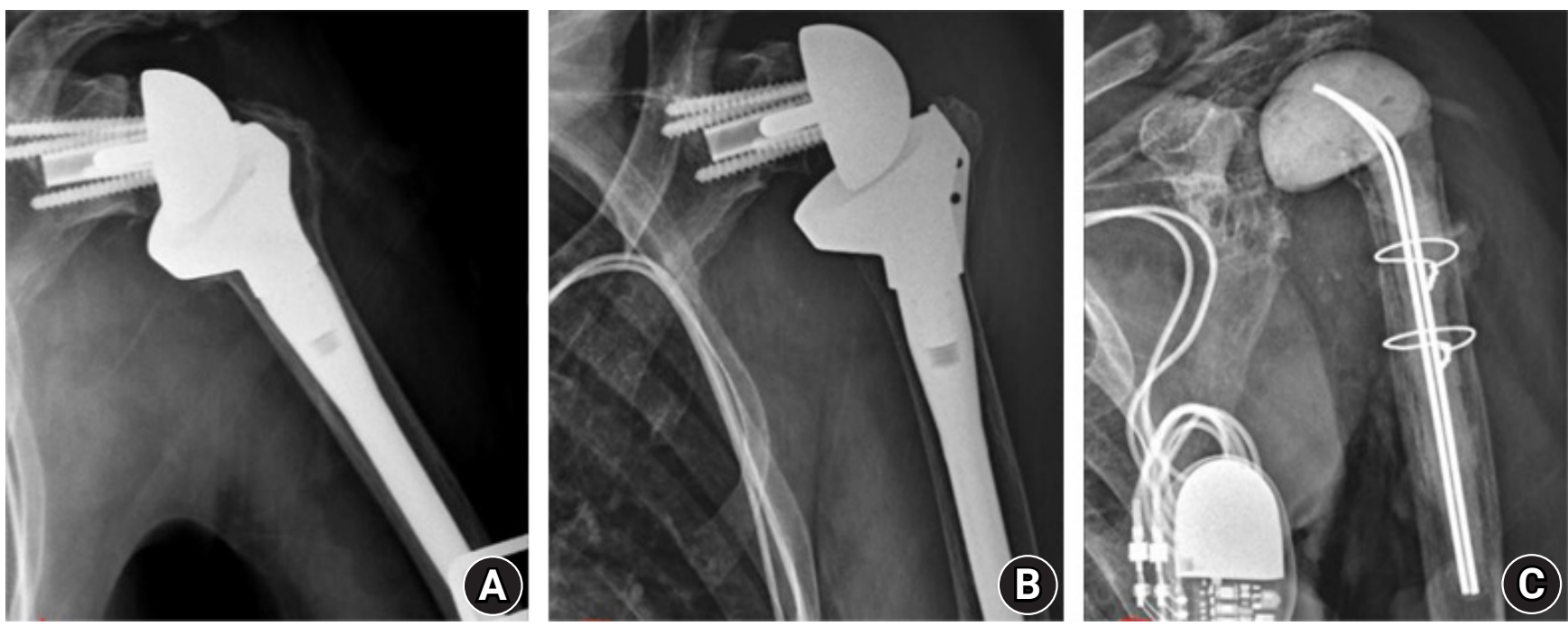

Fig. 5. (A) Immediate postoperative X-ray. (B) Thirty-three-month follow-up with acute onset infection X-ray showing scapular notching with no definite radiologic findings of infection. (C) Three months after prosthesis of antibiotic-loaded acrylic cement insertion. 
lished a comparative study between conventional and BIO-RSA. There was bone graft incorporation in all BIO-RSA with no evidence of graft resorption. However, the BIO-RSA technique was associated with an increase in scapular stress fracture rate compared to the standard RSA (9.1\% in standard RSA and $16.7 \%$ in BIO-RSA); this difference was not significant ( $\mathrm{p}=0.64)$. We also had one case of scapular neck stress fracture that was managed conservatively. In the same study, a statistically significant difference was identified when comparing the rates of scapular notching (standard RSA 68\% vs. BIO-RSA 33\%; p=0.028). Another study by Collin et al. [15] did not demonstrate any advantage of BIO-RSA over conventional RSA including scapular notching. However, their notching rate was low (5\%) compared to our study. Contradictory to this, Athwal et al. [16] reported a significantly higher frequency of scapular notching $(\mathrm{p}=0.022)$ in the RSA cohort compared to the BIO-RSA cohort: $75 \%$ versus $40 \%$, but no other outcome measurements were statistically different, including range of motion, strength, and validated outcome scores in their study. All of their patients had grade I/II notching. In our study, 90\% (18/20) of patients developed grade I/II notching, while the remaining 10\% (2/20) exhibited grade III notching. This high-grade notching is much lower than considered with conventional RSA (>25\%) [2,17].

We believe multiple factors should be consider for notching, including humeral neck shaft angle, inferior overhang of the glenosphere, inferior tilting of the glenosphere, and increasing glenosphere arc $[4,5]$. The reason for scapular notching is usually to achieve contact between the liner and bone at the terminal range of motion, especially for adduction and internal rotations. Bone graft on the glenoid allows relatively greater impingement-free motion, i.e., instead of the curved inferior border of the glenoid, there is a horizontal inferior border of the glenoid after grafting, similar to the long neck scapula. This can increase free range of motion to a certain extent but cannot prevent the liner from contacting the glenoid at the terminal range of motion in adduction and internal rotation. However, further biomechanical studies are required to quantify this hypothesis. Moreover, in the comparison between "notching" and "no-notching" groups, there were no significant differences in demographics, radiological, or clinical outcomes except AT distance $(\mathrm{p}=0.003)$. This AT distance was used to assess the amount of humeral lengthening. The GT distance to assess the amount of lateralization of the humerus exhibited no significant difference between the two groups $(p=0.940)$. We believe that humeral lengthening (increased AT distance) without adequate glenoid lateralization caused the humeral component to impinge inferiorly on the glenoid and potentially lead to increased notching. We typically used 7-mm- thick autografts rather than 1-cm-thick autografts as described in the original technique. In the small body-sized Asian population compared to the Caucasian population, 1-cm-thick autografts can create too much tension during reduction of the prosthesis. However, small glenoid lateralization combined with humeral lengthening could create more notching than expected, and adequate glenoid lateralization with appropriate humeral lengthening could reduce scapular notching.

In addition, higher rates of notching observed in our study compared to other BIO-RSA studies might be because of our study population and prosthesis sizing limitations. As already quantified, the East Asian population has smaller glenoid sizes, especially females $[18,19]$. This reverse prosthesis design was not originally designed to their morphology. All our patients were implanted with $25-\mathrm{mm}$ base plate with $36-\mathrm{mm}$ glenosphere, which was not the case with the previous study. Athwal and Faber [20] reported a $62 \%$ notching rate with $25-\mathrm{mm}$ glenoid base plates (without bony increased offset) in their series; this was not near our rate of notching (60.5\%). Many studies have shown reductions in notching and higher impingement-free adductions with larger glenosphere of diameter of 42 or $44 \mathrm{~mm}$ compared to 36- or 38-mm-diameter glenosphere, which also can explain the notching rate in our study [21-25].

Boileau et al. [17] expanded on the application of BIO-RSA and reported their outcomes in glenoid deficiencies using angled BIO-RSA with trapezoidal grafts. However, in our study, we had three cases of severe glenoid defects, but they were managed with standard humeral head autograft (similar to trapezoidal graft) only without any complications. As an alternative to BIO-RSA, two other lateralization options are available with metallic glenoid lateralization and humerus lateralization. Metallic glenoid lateralization is a similar concept to the lateralized center of rotation. Results as reported by Cuff et al. [26] are encouraging, with reduced rates (9\%) for scapular notching and no glenoid loosening. However, they reported 3\% asymptomatic humeral stem loosening, which is a concern. In addition, Harman et al. [8] reported substantially increased moments (69\%) at the baseplate-bone interface, which possibly could lead to loosening of the baseplate and has been a particular concern with this technique of lateralization. In our study with BIO-RSA, we encountered one humeral stem loosening case. The overall rate of radiolucent lines around the humeral stem in our study was $44.74 \%$ ( $46.66 \%$ for cemented stems vs. $25 \%$ for un-cemented stems). Though our aseptic loosening rate was similar to that of other studies, the finding of radiolucent lines is higher than reported with conventional RSA (15.9\% for cemented stems and 9.5\% for uncemented stems) [27]. 
The introduction of humerus lateralization has been a recent development and has exhibited good results. Franceschetti et al. [28] in their comparative study between BIO-RSA and $145^{\circ}$ onlay curved stems showed similar clinical outcomes with equally reduced rates of scapular notching. Only significant improvement in the BIO-RSA group over the curved stem group was increased external rotation in their study. However, it did not result in improved functional outcomes. The curved humeral stem provides an easier lateralization alternative compared to $\mathrm{BIO}$ RSA. In an analysis of 485 consecutive cases, scapular spine fractures exhibited increased prevalence after the humeral onlay curved short stem design RSA [29]. Long-term comparative studies are needed to prove the superiority of either method.

There were six complications in our series. The first involved one case of heterotopic ossification observed on the X-ray at 1.5 months postoperatively. Verhofste et al. [30] reported a very high incidence (29.5\%) of heterotopic ossification following RSA. Although this incidence is much higher than that in our study, the point of similarity between their study and our study is the timing of heterotopic ossification. They have reported that $81.6 \%$ of heterotopic ossification developed within the first three months postoperatively, similar to our findings. Also, in our study, patients with heterotopic ossification did not have an altered functional outcome compared to other patients, similar to their findings.

The second complication was inferior screw malposition, also observed in one case. The screw was from the inferior aspect, directed inferior to the inferior border of the glenoid and lateral border of the scapula. However, no glenoid loosening was observed at the mid-term follow-up. This was a technical complication, and we now prefer an almost horizontal direction of the screw especially in long neck scapula patients.

The third complication was intraoperative humerus metaphyseal fracture in three patients and was treated with circumferential cerclage wiring. None of the patients developed subsidence of the stem. However, gradual resorption of bone was noted at the medial calcar area and GT area without any signs of subsidence/ loosening on subsequent X-rays.

As already mentioned previously, we noted one case of humeral stem loosening in one of the patients with a cemented stem without any signs of infection. The patient complained of occasional pain (VAS 2). Upon examination, there was restriction of motion (forward flexion 110, abduction 110, external rotation 20, and internal rotation L4) and in the radiographs, lysis around the humeral stem with rotation. Infection was ruled out, and the patient was advised to undergo revision of the stem to further improve the clinical outcome. However, because of minimal complaints from the patient and their advanced age (80 years), she did not

want to undergo the revision operation. This case was similar to others with the aseptic loosening rate $(1.18 \%)$ observed with the cemented humeral stem as reported by Gilot et al. [3].

The fifth complication was one case of late infection developing 33 months after surgery. The patient was treated with PROSTALAC (antibiotic impregnated cement) insertion, and has yet to receive revision prosthesis surgery. The patient is 1 year postoperative after PROSTALAC insertion with healing of the wound and was unable to receive revision surgery due to aggravated comorbidities (pacemaker and age 91 years at present, the oldest patient in our study). As reported in the literature, functional outcomes of infection patients are usually poorer than those of primary RSA [31], and the same was found in our study. The sixth complication as described previously was a non-traumatic scapula fracture which developed after 27 months of the primary procedure. The fracture occurred at the site medial to the tip of the glenoid screws and was minimally displaced. Kirzner et al. [14] reported significantly higher scapula stress fractures with BIO-RSA compared to conventional RSA. However, our rate was much lower than that of their series (2.6\% vs. $16.7 \%)$, and this complication must be considered while using BIO-RSA. The patient was managed conservatively with an arm sling for 6 weeks. Three-month X-ray revealed callus formation with no impact on the functional outcome.

There are several shortcomings of our study. First, we did not include a cohort against which to compare our data, limiting analysis of the particular method for a definite conclusion. Second, we relied on X-rays to assess autograft incorporation rather than performing postoperative CT scans in every patient. However, artifacts can obscure postoperative CT. Third, the study design was retrospective, and the number of patients (38 patients) was too small and underwent only a minimum of 2 years of follow-up.

BIO-RSA is a promising modification in the conventional RSA design, showing improved clinical outcomes with similar rates of complications in the short-term compared to conventional RSA. However, we observed higher rates of scapular notching than reported in a previous series with no impact on functional outcome. In addition, adequate glenoid lateralization with appropriate humeral lengthening could reduce scapular notching. More long-term comparative studies with newer concepts like humeral lateralization must be conducted to adequately prove the superiority of either method.

\section{ORCID}

Kirtan Tankshali

Dong-Whan Suh https://orcid.org/0000-0003-0734-3316

https://orcid.org/0000-0003-3029-2553 
Jong-Hun Ji

Chang-Yeon Kim https://orcid.org/0000-0003-1087-3254

https://orcid.org/0000-0001-5880-4747

\section{REFERENCES}

1. Flatow EL, Harrison AK. A history of reverse total shoulder arthroplasty. Clin Orthop Relat Res 2011;469:2432-9.

2. Boileau P, Watkinson D, Hatzidakis AM, Hovorka I. Neer Award 2005: The Grammont reverse shoulder prosthesis: results in cuff tear arthritis, fracture sequelae, and revision arthroplasty. J Shoulder Elbow Surg 2006;15:527-40.

3. Gilot G, Alvarez-Pinzon AM, Wright TW, et al. The incidence of radiographic aseptic loosening of the humeral component in reverse total shoulder arthroplasty. J Shoulder Elbow Surg 2015;24:1555-9.

4. Gutiérrez S, Comiskey CA 4th, Luo ZP, Pupello DR, Frankle MA. Range of impingement-free abduction and adduction deficit after reverse shoulder arthroplasty: hierarchy of surgical and implant-design-related factors. J Bone Joint Surg Am 2008;90:2606-15.

5. Huri G, Familiari F, Salari N, Petersen SA, Doral MN, McFarland EG. Prosthetic design of reverse shoulder arthroplasty contributes to scapular notching and instability. World J Orthop 2016;7:738-45.

6. Chae SW, Lee H, Kim SM, Lee J, Han SH, Kim SY. Primary stability of inferior tilt fixation of the glenoid component in reverse total shoulder arthroplasty: a finite element study. J Orthop Res 2016;34:1061-8.

7. Tashjian RZ, Burks RT, Zhang Y, Henninger HB. Reverse total shoulder arthroplasty: a biomechanical evaluation of humeral and glenosphere hardware configuration. J Shoulder Elbow Surg 2015;24:e68-77.

8. Harman M, Frankle M, Vasey M, Banks S. Initial glenoid component fixation in "reverse" total shoulder arthroplasty: a biomechanical evaluation. J Shoulder Elbow Surg 2005;14(1 Suppl S):162S-167S.

9. Boileau P, Moineau G, Roussanne Y, O’Shea K. Bony increased-offset reversed shoulder arthroplasty: minimizing scapular impingement while maximizing glenoid fixation. Clin Orthop Relat Res 2011;469:2558-67.

10. Walch G, Badet R, Boulahia A, Khoury A. Morphologic study of the glenoid in primary glenohumeral osteoarthritis. J Arthroplasty 1999;14:756-60.

11. Hamada K, Fukuda H, Mikasa M, Kobayashi Y. Roentgenographic findings in massive rotator cuff tears: a long-term observation. Clin Orthop Relat Res 1990;(254):92-6.

12. Boileau P, Watkinson DJ, Hatzidakis AM, Balg F. Grammont re- verse prosthesis: design, rationale, and biomechanics. J Shoulder Elbow Surg 2005;14(1 Suppl S):147S-161S.

13. Sirveaux F, Favard L, Oudet D, Huquet D, Walch G, Molé D. Grammont inverted total shoulder arthroplasty in the treatment of glenohumeral osteoarthritis with massive rupture of the cuff: results of a multicentre study of 80 shoulders. J Bone Joint Surg Br 2004;86:388-95.

14. Kirzner N, Paul E, Moaveni A. Reverse shoulder arthroplasty vs BIO-RSA: clinical and radiographic outcomes at short term follow-up. J Orthop Surg Res 2018;13:256.

15. Collin P, Liu X, Denard PJ, Gain S, Nowak A, Lädermann A. Standard versus bony increased-offset reverse shoulder arthroplasty: a retrospective comparative cohort study. J Shoulder Elbow Surg 2018;27:59-64.

16. Athwal GS, MacDermid JC, Reddy KM, Marsh JP, Faber KJ, Drosdowech D. Does bony increased-offset reverse shoulder arthroplasty decrease scapular notching. J Shoulder Elbow Surg 2015;24:468-73.

17. Boileau P, Morin-Salvo N, Gauci MO, Seeto BL, Chalmers PN, Holzer N, et al. Angled BIO-RSA (bony-increased offset-reverse shoulder arthroplasty): a solution for the management of glenoid bone loss and erosion. J Shoulder Elbow Surg 2017; 26:2133-42.

18. Cabezas AF, Krebes K, Hussey MM, et al. Morphologic variability of the shoulder between the populations of North American and East Asian. Clin Orthop Surg 2016;8:280-7.

19. Ji JH, Jeong JY, Song HS, Ok JH, Yang SJ, Jeon BK, et al. Early clinical results of reverse total shoulder arthroplasty in the Korean population. J Shoulder Elbow Surg 2013;22:1102-7.

20. Athwal GS, Faber KJ. Outcomes of reverse shoulder arthroplasty using a mini 25-mm glenoid baseplate. Int Orthop 2016;40:10913.

21. de Wilde LF, Poncet D, Middernacht B, Ekelund A. Prosthetic overhang is the most effective way to prevent scapular conflict in a reverse total shoulder prosthesis. Acta Orthop 2010;81:71926.

22. Friedman RJ, Barcel DA, Eichinger JK. Scapular notching in reverse total shoulder arthroplasty. J Am Acad Orthop Surg 2019; 27:200-9.

23. Nyffeler RW, Werner CM, Gerber C. Biomechanical relevance of glenoid component positioning in the reverse Delta III total shoulder prosthesis. J Shoulder Elbow Surg 2005;14:524-8.

24. Torrens C, Guirro P, Miquel J, Santana F. Influence of glenosphere size on the development of scapular notching: a prospective randomized study. J Shoulder Elbow Surg 2016;25:1735-41.

25. Werner BS, Chaoui J, Walch G. Glenosphere design affects range of movement and risk of friction-type scapular impinge- 
ment in reverse shoulder arthroplasty. Bone Joint J 2018;100: 1182-6.

26. Cuff D, Clark R, Pupello D, Frankle M. Reverse shoulder arthroplasty for the treatment of rotator cuff deficiency: a concise follow-up, at a minimum of five years, of a previous report. J Bone Joint Surg Am 2012;94:1996-2000.

27. Grey B, Rodseth RN, Roche SJ. Humeral stem loosening following reverse shoulder arthroplasty: a systematic review and meta-analysis. JBJS Rev 2018;6:e5.

28. Franceschetti E, Ranieri R, Giovanetti de Sanctis E, Palumbo A, Franceschi F. Clinical results of bony increased-offset reverse shoulder arthroplasty (BIO-RSA) associated with an onlay $145^{\circ}$ curved stem in patients with cuff tear arthropathy: a compara- tive study. J Shoulder Elbow Surg 2020;29:58-67.

29. Ascione F, Kilian CM, Laughlin MS, et al. Increased scapular spine fractures after reverse shoulder arthroplasty with a humeral onlay short stem: an analysis of 485 consecutive cases. J Shoulder Elbow Surg 2018;27:2183-90.

30. Verhofste B, Decock T, Van Tongel A, De Wilde L. Heterotopic ossification after reverse total shoulder arthroplasty. Bone Joint J 2016;98:1215-21.

31. Jacquot A, Sirveaux F, Roche O, Favard L, Clavert P, Molé D. Surgical management of the infected reversed shoulder arthroplasty: a French multicenter study of reoperation in 32 patients. J Shoulder Elbow Surg 2015;24:1713-22. 